\title{
Human blood and synovial fluid neutrophils cultured in vitro undergo programmed cell death which is promoted by the addition of synovial fluid
}

\author{
A L Bell, M K Magill, R McKane, A E Irvine
}

\begin{abstract}
Objective-To assess the influence of inflammatory synovial fluid (SF) on apoptosis of joint and blood neutrophils with particular reference to levels of colony stimulating factors (CSF) contained therein.

Methods-Neutrophils were separated from fresh synovial fluid and from peripheral blood by density gradient centrifugation. Apoptosis was assayed by light microscope morphology and DNA degradation. CSFs were assayed using bone marrow bioassay and enzyme linked immunosorbent assays for granulocyte (G-) and granulocyte macrophage (GM-) CSF. Separated neutrophils were cultured in vitro and exposed to: varying concentrations of SF in which CSF levels were measured, recombinant G-CSF and GM-CSF, and hyaluronic acid control solutions. Numbers of apoptotic neutrophils and CSF levels were also measured in fresh SF samples.
\end{abstract}

Results-The addition of autologous or heterologous inflammatory SF to blood or joint cavity neutrophils cultured in vitro caused a significant dose dependent increase in the percentage of cells becoming apoptotic with time as measured morphologically and confirmed by DNA degradation. The effect bore no relationship to levels of CSF in joint fluid, despite our finding that GM-CSF produced inhibition of neutrophil apoptosis in vitro.

Conclusion-These data suggest that SF contains a factor or factors capable of directly or indirectly promoting neutrophil apoptosis and normally powerful enough to overcome the apoptosis inhibiting effects of cytokines such as GM-CSF at concentrations usually found in inflammatory synovial fluids.

(Ann Rheum Dis 1995; 54: 910-915)

Inflammation research hitherto has tended to focus on initiating lesions, the events surrounding white cell influx to a site of tissue injury, and the amplifying effects of a large variety of mediators. By contrast, few ${ }^{1}$ have directed attention to factors which govern resolution of inflammatory reactions, despite the often expressed view that chronic or persistent inflammation results from an improperly resolved acute inflammatory reaction. In chronic inflammatory arthritis, such as rheumatoid disease, large numbers of neutrophils are attracted across the synovial membrane from the general circulation, congregate within the joint, and become activated. ${ }^{2}$ If these neutrophils are not efficiently disposed of, their production of inflammatory mediatorsincluding cytokines, prostaglandins, proteolytic enzymes, and reactive oxygen species-could prolong the inflammatory reaction, leading to further tissue destruction. Their removal would appear to be of considerable importance in the resolution of inflammation.

The fate of neutrophils in the inflamed joint is unclear: there is no evidence that they reenter the circulation, and it has often been assumed that they disappear by a non-specific process of necrosis. Recently, however, neutrophils exhibiting morphological features of apoptotic cell death have been described in pathological joint fluids. ${ }^{34}$ Savill et al ${ }^{3}$ showed that neutrophils from inflammatory synovial fluid cultured in vitro undergo morphological and biochemical changes characteristic of apoptosis; they also showed a close relationship between increasing neutrophil apoptosis and the degree of macrophage recognition and subsequent engulfment of these aging neutrophils. ${ }^{3}$ This observation explains the presence in pathological joint fluid of neutrophil laden cytophagocytic macrophages (CPM)-cells initially described as specific for Reiter's disease, ${ }^{5}$ though this was not supported by more recent work. ${ }^{46}$ Accurate cytological assessment of CPMs in relation to other cells in inflammatory synovial fluid provides a histological basis for the proposal that clearance of effete neutrophils from the inflamed synovial cavity depends on their first becoming apoptotic. $^{34}$

Several reports have demonstrated the presence of cytokine colony stimulating factors (CSFs) in synovial fluids ${ }^{7-11}$ and highlighted their potential role in the pathogenesis of inflammatory arthritis. ${ }^{7910}$ CSFs have the capacity to promote cell survival by suppression of apoptosis ${ }^{12}$ - an effect seen in neutrophils treated with granulocyte (G-) ${ }^{13}$ and granulocyte macrophage (GM-) $)^{13-15}$ CSF. Such an effect on polymorphonuclear cells within the inflamed joint cavity is likely to perpetuate both acute and chronic synovitis. The effect of other cytokines present in inflammatory joint fluid is less certain; for example, both interleukin- 6 and tumour necrosis factor $\alpha$ have been shown to enhance ${ }^{16}$ or inhibit $^{13}$ neutrophil apoptosis in vitro. 
We tested the hypothesis that synovial fluid from patients with inflammatory joint disease may prolong neutrophil survival and activity by suppressing apoptosis as a result of CSFs contained therein, thus contributing to persistent and damaging inflammatory synovitis.

\section{Materials and methods \\ PATIENTS}

Synovial fluid was obtained from patients with a variety of inflammatory arthritides, after aspiration required as part of routine clinical treatment at joint injection clinics in the Royal Victoria, Musgrave Park, and Belfast City hospitals. Patients were classified by diagnosis according to accepted criteria.

\section{PREPARATION OF FRESH SYNOVIAL FLUID SAMPLES}

Samples were centrifuged at $400 \mathrm{~g}$ for 20 minutes. The fluid was removed by aspiration and used directly in culture experiments, stained for apoptosis or stored in $1 \mathrm{ml}$ aliquots at $-20^{\circ} \mathrm{C}$ until required for subsequent use. Cytospins of synovial fluid were prepared within three hours of aspiration and stained for morphological assessment of apoptosis as described below. At least 500 cells in each preparation were counted under high power view.

\section{PREPARATION OF SYNOVIAL FLUID}

NEUTROPHILS

Synovial fluid cells were resuspended in RPMI 1640 medium (Gibco) with $10 \%$ fetal calf serum (FCS) (Globepharm) and separated over Ficoll (Pharmacia) at $400 \mathrm{~g}$ for 20 minutes. The cell pellet was washed twice with phosphate buffered serum (PBS), and the cells resuspended in an appropriate volume of RPMI 1640-10\% FCS and counted using a haemocytometer. Preparations contained $>90 \%$ neutrophils.

PREPARATION OF BLOOD NEUTROPHILS

Neutrophils were isolated from heparinised peripheral blood from patients and from normal consenting healthy volunteer donors. A double gradient was formed by layering an equal volume of Ficoll ( $3 \mathrm{ml}$ ) over Histopaque1119 (3 ml) (Sigma). Whole blood (6 ml) was carefully layered onto the upper Ficoll layer. The tubes were centrifuged at $700 \mathrm{~g}$ for 30 minutes at room temperature. Cells of the granulocyte series were found at the Ficolly 1119 interphase. ${ }^{18}$ The lower granulocyte layer was removed and washed twice with PBS. The cells were then resuspended in an appropriate volume of RPMI 1640 medium with 10\% FCS and counted using a haemocytometer. Preparations contained $>90 \%$ neutrophils.

CULTURE OF NEUTROPHILS

Neutrophils prepared as described above were cultured at $1 \times 10^{6}$ cells per $\mathrm{ml}$ in RPMI
$1640-10 \% \mathrm{FCS}$ in $5 \%$ carbon dioxide at $37^{\circ} \mathrm{C}$. Cultures were performed in $35 \mathrm{~mm}^{3}$ plastic petri dishes and cells prepared for assay as described below. Cell recovery was not affected by the addition of synovial fluid. The effects of co-culture with the following substances was examined:

(1) varying concentrations of autologous and heterologous synovial fluid $(0,25,50,75 \%)$;

(2) recombinant CSFs, G-CSF (Amgen) 2000

$\mathrm{pg} / \mathrm{ml}$ and GM-CSF (Amgen) 100, 500, 2000

$\mathrm{pg} / \mathrm{ml}$ (concentration ranges found in inflammatory joint fluid ${ }^{11}$ );

(3) purified hyaluronic acid (Sigma) in final concentrations of $0 \%, 25 \%(1.25 \mathrm{mg} / \mathrm{ml}), 50 \%$ $(2.5 \mathrm{mg} / \mathrm{ml})$ in RPMI 1640 .

MEASUREMENTS OF APOPTOSIS

The degree of neutrophil apoptosis in each culture was assessed by both DNA fragmentation and morphology.

DNA fragmentation assay- $1 \times 10^{6}$ cells per test point were centrifuged in a $1.5 \mathrm{ml}$ microfuge tube for 10 minutes at $1300 \mathrm{rpm}$. They were resuspended by vortexing in $20 \mu \mathrm{l}$ of $10 \mathrm{mmol} / 1$ EDTA $\mathrm{pH} 8.0,50 \mathrm{mmol} / 1$ Tris- $\mathrm{HCl} \mathrm{pH} 8.0,0.5 \% \mathrm{w} / \mathrm{v}$ sodium lauryl sarkosinate and $0.5 \mathrm{mg} / \mathrm{ml}$ proteinase $\mathrm{K}$, and incubated at $50^{\circ} \mathrm{C}$ for one hour. RNase A was added $(10 \mu \mathrm{l}$ of $0.5 \mathrm{mg} / \mathrm{ml})$ and the sample heated at $50^{\circ} \mathrm{C}$ for a further one hour. Immediately before it was loaded on the gel, the sample was heated to $70^{\circ} \mathrm{C}$ and $10 \mu \mathrm{l}$ of $10 \mathrm{mmol} / 1 \mathrm{EDTA}, 1 \%$ low melting agarose, $0.25 \%$ bromophenol blue, and $40 \%$ sucrose was added. Samples were loaded onto the dry wells of a $2 \%$ agarose gel; electrophoresis was carried out in $\mathrm{xl}$ Tris acetate EDTA (50 $\mathrm{mmol} / 1$ Tris acetate, $\mathrm{pH} 8 \cdot 3-1 \mathrm{mmol} / 1$ EDTA) at $6 \mathrm{~V} / \mathrm{cm}$ until the marker dye had run 3-4 cm. Lambda phage DNA digested with Hind III, and DNA prepared from mouse thymocytes which had been aged for 24 hours in RPMI 1640 with $10 \%$ FCS were included as molecular weight markers and positive controls, respectively.

Morphology-Cytospins were prepared in duplicate at $1 \times 10^{5}$ cells per slide for each test culture and those prepared within three hours of aspiration on 39 separate synovial fluid samples were studied. Slides were stained with Hema-Tek modified Wright stain and apoptotic polymorphs counted under high power view in at least 500 cells. Neutrophils were considered apoptotic if they exhibited the highly characteristic morphological features of chromatin aggregation, nuclear pyknosis, and cytoplasmic vacuolation described and validated previously ${ }^{3}$ (fig 1).

\section{CSF LEVELS}

The concentration of CSFs in a number of the synovial fluids used for the culture experiments was measured by a bone marrow colony forming bioassay ${ }^{19}$ and also by enzyme linked immunosorbent assay (ELISA) for GM-CSF and G-CSF (Quantikine, British Biotechnology Ltd). ELISA sensitivities were $10.9 \mathrm{pg} / \mathrm{ml}$ 


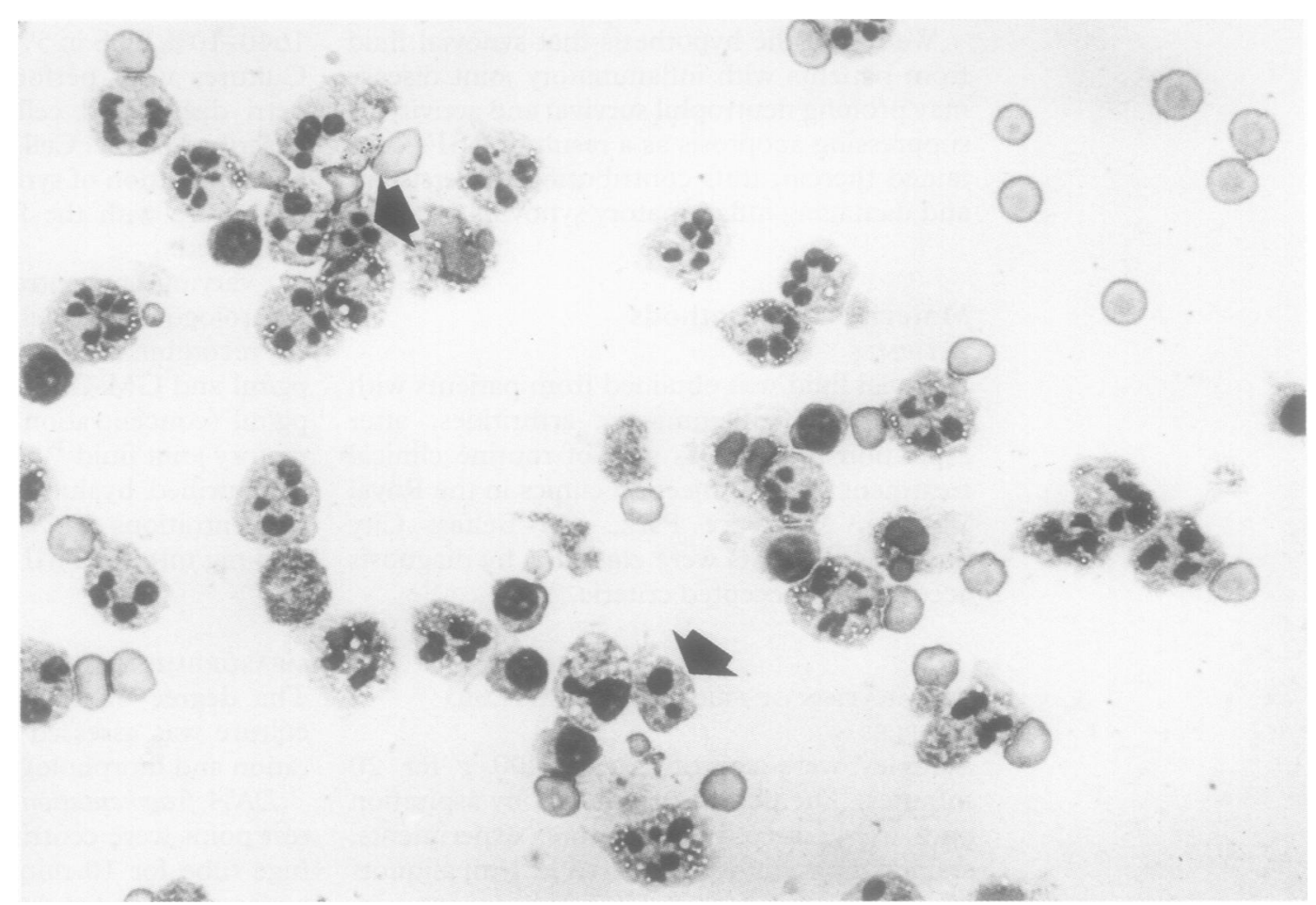

Figure 1 Separated synovial neutrophils after 24 hours in vitro culture in $10 \%$ synovial fluid. Cells showing typical morphological features of apoptosis are arrowed. Modified Wright stain; original magnification $\times 400$.

for G-CSF and $2 \cdot 8 \mathrm{pg} / \mathrm{ml}$ for GM-CSF. These levels were correlated to the degree of neutrophil apoptosis.

\section{STATISTICAL ANALYSIS}

Non-parametric tests of between group differences were applied throughout. Correlations were assessed by Spearman's rank test.

\section{Results}

ANALYSIS OF FRESH SYNOVIAL FLUID SAMPLES Apoptotic polymorph counts-Apoptotic polymorphs were found in 26 fluids including 19 of 28 rheumatoid arthritis (RA) samples, five of five other inflammatory arthritis samples (three psoriatic arthritis, one reactive arthritis, one gout), and two of four osteoarthritis (OA) samples. They comprised $0-22 \%$ of cells (mean 1.32, median 0.5, SEM 0.5), and were significantly more frequent in seropositive than seronegative RA $(\mathrm{p}<0.05, \mathrm{n}=28$, Mann Whitney $U$ test). Percentages of apoptotic polymorphs were significantly reduced in patients currently receiving steroid treatment ( $\mathrm{n}=9$, mean 0.22, SEM 0.09) compared with those not taking steroids $(n=22$, mean $1 \cdot 1$, SEM 0.2; $\mathrm{p}<0.02$ Mann Whitney $U$ test). There was no correlation between neutrophil count in the fluid and percentage of apoptotic cells. No relationship was found between apoptotic cell percentage and erythrocyte sedimentation rate, serum or synovial fluid $C$ reactive protein, or disease activity (patient global score on $0-3$ scale).

Colony stimulating factor assays-GM-CSF estimations were available for 19 of the above synovial fluid samples, but there were no significant differences in numbers of apoptotic polymorphs between samples in which
GM-CSF was found in significant quantities (5-239 $\mathrm{pg} / \mathrm{ml}, \mathrm{n}=11$ ) and those in which GM-CSF was not found $(0-4 \mathrm{pg} / \mathrm{ml}, \mathrm{n}=18)$; mean numbers of apoptotic cells $1 \cdot 14$ (SEM 0.42) and 1.81 (SEM 1.1), respectively. Mean numbers of apoptotic cells were $1 \cdot 1$ (SEM 0.28) in G-CSF positive $(14-800 \mathrm{pg} / \mathrm{ml}$, $\mathrm{n}=20$ ) and 0.58 (SEM 0.16) in G-CSF negative ( $0 \mathrm{pg} / \mathrm{ml}, \mathrm{n}=13)$ samples (NS). Similarly, in 28 samples CSF levels were estimated by bioassay and there were no significant differences in levels of apoptotic polymorphs between samples that were bioassay positive $(n=18$, mean $0.994 ; 0.302$ SEM) and those that were negative $(n=10$, mean $2 \cdot 71$; SEM 2・16).

\section{NEUTROPHILS AGED IN CULTURE}

Synovial fluid neutrophils-The table summarises the results. These show that cultured synovial fluid polymorphs become significantly apoptotic after 24 hours (median $46 \%$ apoptotic polymorphs) and 48 hours (median $67 \%$ apoptotic polymorphs) compared with baseline values (median $2 \cdot 4 \%$ ). There was no significant loss of viability at 24 hours (6\% trypan

Effect of aging in culture and addition of autologous synovial fluid on apoptosis of synovial polymorphs

\begin{tabular}{lllll}
\hline \multicolumn{5}{c}{ Apoptotic neutrophils (\%) } \\
\cline { 2 - 5 } & $0 \% S F$ & $25 \% S F$ & $50 \% S F$ & $75 \% S F$ \\
\hline Baseline & $2 \cdot 4$ & & & \\
& $4 \cdot 3(1 \cdot 2)$ & & & \\
24 h cultures & $46 \star \star \star$ & 49 & $70+t \dagger$ & $91+\mathrm{t}$ \\
& $40(6 \cdot 1)$ & $47 \cdot 2(2 \cdot 7)$ & $73(4 \cdot 0)$ & $87 \cdot 6(3 \cdot 8)$ \\
48 h cultures & $67 \star \star \star$ & 61 & $81+t+$ & $99 \cdot 5+t \dagger$ \\
& $57 \cdot 7(12 \cdot 3)$ & $60(7 \cdot 2)$ & $69 \cdot 3(9 \cdot 1)$ & $95 \cdot 5(2 \cdot 3)$ \\
\hline
\end{tabular}

Values are median and mean (SEM) for nine separate

$\mathrm{SF}=$ Synovial fluid. $\mathrm{p}<0.001$ compared with: ${ }^{\star \star \star}$ baseline; $\mathrm{t}+\mathrm{t} 0 \%$ synovial fluid. 
blue positive cells compared with mean $5 \%$ at baseline).

Effect of added autologous synovial fluidSynovial fluid added in increasing aliquots of $25 \%, 50 \%$, and $75 \%$ replacement for RPMI medium produced a dose dependent enhancement of the effect of aging on apoptosis: significant $(p<0.001)$ enhancement of apoptosis was seen in the $50 \%$ and $75 \%$ added synovial fluid cultures (table). In these experiments autologous synovial fluid was used (five RA, two other inflammatory arthritis, and two OA). There was no significant loss of viability after 24 hours in culture (4\% trypan blue positive cells), but at 48 hours an unacceptable degree of necrosis was evident (mean 13\% trypan blue positive).

Chromatin degradation - Chromatin gels read by a blinded observer revealed 'stepladder' degeneration patterns typical of apoptosis, confirming the results of morphological assessments in all cases (fig 2).

CSFs and apoptosis of aging neutrophilsLevels of GM-CSF and G-CSF in the added fluid samples bore no relationship to their capacity to promote neutrophil apoptosis: correlation coefficients were all less than $r=0 \cdot 2$ (Spearman rank correlation). By chance, GM-CSF concentrations in the samples used in the table (and figure 3) were less than $100 \mathrm{pg} / \mathrm{ml}$ and therefore a strong inhibitory effect on neutrophil apoptosis would not be expected (see below); nevertheless, of the samples used above, those with GM-CSF levels in the $70 \mathrm{pg} / \mathrm{ml}$ range were able to promote 24 hour apoptosis of cells to as much as $100 \%$ compared with $45 \%$ in untreated samples (patient ML: RA). In contrast, some fluids in which GM-CSF was absent produced a more modest effect ( $79 \%$ compared with $41 \%$ untreated: patient DW, psoriatic arthritis). In other experiments (see below), fluids with GM-CSF levels well into the inhibitory range were not associated with inhibition of the rate of neutrophil apoptosis.

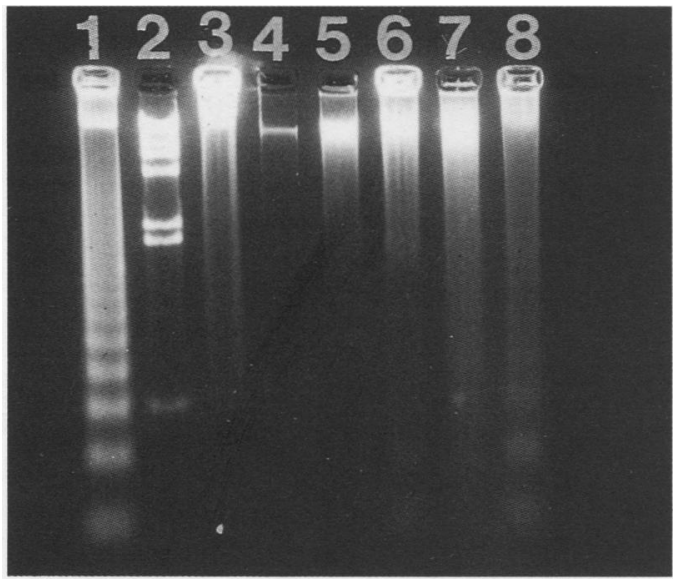

Figure 2 Sample gel electrophoresis showing 'stepladder' DNA degradation typical of apoptosis. LANE 1: (left) control DNA from mouse thymus cells aged for 24 hours, LANE 2: $\lambda$-phage DNA digested with Hind-III. Synovial neutrophil DNA from patient with psoriatic arthritisLANE 3: baseline; LANE 4: 24 hour culture in 0\% synovial fluid (SF); LANE 5: 24 hour culture in 50\% SF; LANE 6: 24 hour culture $100 \%$ SF; LANE 7: 48 hour culture in $50 \%$ $S F$; LANE 8: 48 hour culture in $100 \% S F$
Effect of added autologous and heterologous synovial fluid on apoptosis of peripheral blood neutrophils from patients with arthritis-Figure 3 shows the results of the experiments in which peripheral blood neutrophils from five individual patients were exposed to $50 \%$ autologous synovial fluid or $50 \%$ heterologous synovial fluids (from two patients with RA, two patients with psoriatic arthritis, and one with OA). Peripheral blood neutrophils aged in culture without synovial fluid undergo significant levels of apoptosis and this was promoted by the addition of autologous synovial fluid and (to a lesser extent) by heterologous synovial fluid. The mean $\%$ apoptosis of these untreated blood neutrophils $(20 \cdot 4 \%)$ was rather less than that for synovial fluid neutrophils shown in the table $(40 \%)$.

Effect of synovial fluid on promotion of apoptosis of normal peripheral blood neutrophils-Figure 4 shows the effects of synovial fluid $(25 \%)$ from 19 donors (five OA, six RA, and eight other inflammatory arthritides: five psoriatic arthritis, one gout, one reactive arthritis, one undiagnosed chronic knee synovitis), or control medium, on cultures of peripheral blood neutrophils from normal subjects. At 24 hours, normal peripheral blood neutrophils aged without synovial fluid showed a mean of $33 \%$ apoptosis (median $27 \%$, SEM 4.4 ), compared with a mean of $58 \%$ (median $58 \%$, SEM 6.2 ) in the cultures with added synovial fluid. The figure shows that normal blood neutrophils were susceptible to the apoptosis promoting effects of synovial fluids of various diagnostic categories. It also demonstrates variability in apoptosis promotion between samples: in four cases (two RA and two psoriatic arthritis) no apoptosis promoting effect was seen. (In two additional cultures $100 \%$ apoptosis was seen at

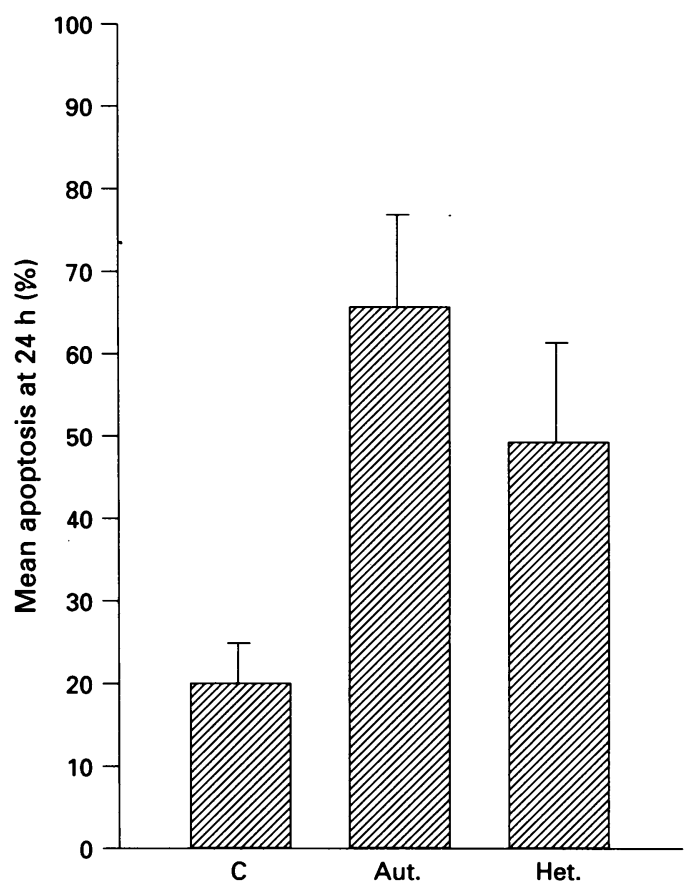

Figure 3 Effect of $50 \%$ autologous (Aut.) and heterologous (Het.) synovial fluid on apoptosis of peripheral blood neutrophils from patients with arthritis. Mean (SEM) of samples from five patients (two rheumatoid arthritis, two psoriatic arthtitis, one osteoarthritis). $C=$ Control (no synovial fiuid). 


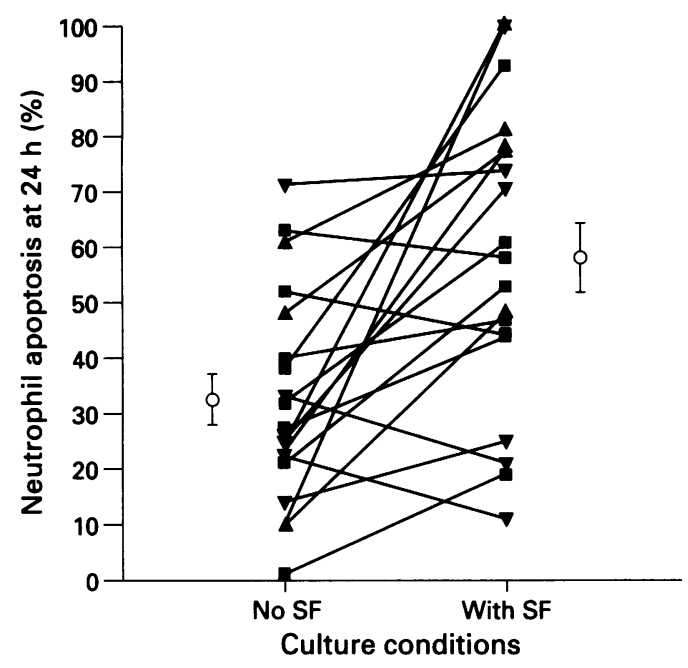

Figure 4 Effect of synovial fluid (SF) on apoptosis of normal peripheral blood neutrophils: rates for individual experiments and mean (SEM error bar) for the two groups. $n=19$ fluid samples used in separate experiments. $\Delta=$ Osteoarthritis; $\mathbf{\square}=$ rheumatoid arthritis; $\boldsymbol{\nabla}=$ other inflammatory arthritides.

24 hours with or without synovial fluid and these were therefore not included in the analysis of synovial fluid effect).

Effect of recombinant cytokines-GM-CSF caused dose dependent non-linear inhibition of peripheral blood and synovial neutrophil apoptosis (fig 5). G-CSF produced no significant effect on neutrophil apoptosis in 24 hour cultures $(\mathrm{n}=7)$.

Effect of hyaluronic acid-Hyaluronic acid (Sigma), even at $2.5 \mathrm{mg} / \mathrm{ml}$, produced no changes in rates of apoptosis in cultured synovial fluid neutrophils $(n=4)$.

\section{Discussion}

In these studies we examined the hypothesis that persistent inflammatory responses in arthritis may result from inhibition of neutrophil apoptosis by cytokine factors, notably CSFs, in synovial fluid. In fresh synovial fluid samples, small percentages of apoptotic neutrophils were identified, but their frequency was not related to CSF levels found within the sample.

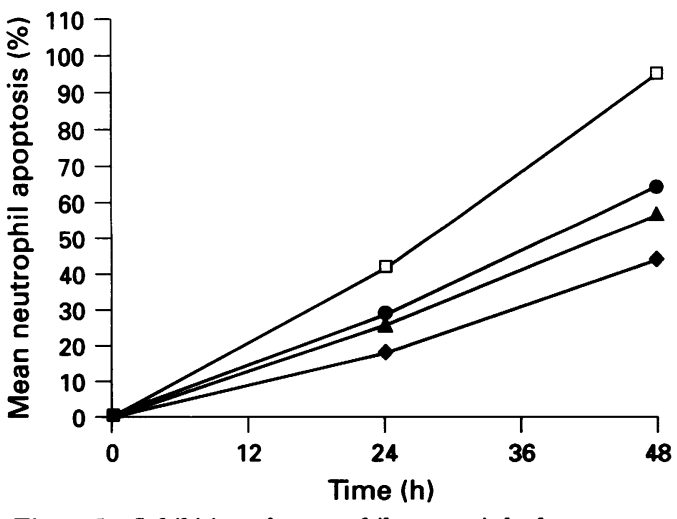

Figure 5 Inhibition of neutrophil apoptosis by human recombinant granulocyte macrophage colony stimulating factor (GM-CSF): mean apoptosis rates at 24 and 48 hours for five individual experiments using cells from separate donors (two synovial fluid, three peripheral blood). Responses were similar in blood and synovial fluid cells. $\square=$ Untreated; $O=100 \mathrm{pg} / \mathrm{ml} ; \Delta=500 \mathrm{pg} / \mathrm{ml} ; \Delta=2000$ $p g / m l$.
Furthermore, we found little association between percent apoptotic neutrophils and clinical diagnosis - a finding in accord with a recent large survey of synovial fluid cytology. ${ }^{20}$ In view of the recognised apoptosis inducing effect of steroids on lymphoid cells, it is of potential interest that significantly fewer apoptotic neutrophils were present in samples from steroid treated patients, though a variety of direct and indirect actions may have produced this effect: cultured myeloid precursors (human promyelocytic leukaemia cell line 60) treated with dexamethasone $10^{-6}-10^{-4} \mathrm{~mol} / \mathrm{l}$ ) in vitro have been found to undergo no change in rate of apoptosis (unpublished data). Differences in samples from steroid treated patients cannot be explained by reference to clinical disease activity.

In order to examine our hypothesis in a more sensitive system, we induced apoptosis in neutrophils from synovial fluid and peripheral blood aged in culture and studied the effects of added synovial fluid and recombinant cytokines. We have shown, in confirmation of the findings of Savill et $a l^{3}$ that cultured synovial and blood neutrophils became increasingly apoptotic with time in culture. Unexpectedly, we found that pathological synovial fluid promotes this effect in a dose and time dependent manner. The effect was independent of the concentration or type (G- or GM-) of CSF contained therein and of the source of the target neutrophil, as cells of both autologous and heterologous synovial fluid, and peripheral blood origin were susceptible. The effect of synovial fluid occurred with both heterologous and autologous samples. Human recombinant GM-CSF was found to inhibit blood neutrophil apoptosis, as reported by others; ${ }^{13} 15$ synovial fluid neutrophils were not resistant to this effect. In contrast, G-CSF at concentrations described in synovial fluid ${ }^{11}$ produced no effect on blood or synovial neutrophil apoptosis in vitro. This finding accords with the data of Brach et al, ${ }^{15}$ but is at variance with the work of others ${ }^{13}$ using human blood neutrophils. We conclude that pathological synovial fluid promotes neutrophil apoptosis despite the presence of potent inhibitors such as GM-CSF. Our data do not exclude the possibility that high concentrations of GM-CSF in synovial fluid could overcome its usual apoptosis accelerating effect, though we have documented no such occurrence.

In the neutrophil, the intracellular machinery driving apoptosis appears quite distinct from that of many other cell types. For example, neutrophil apoptosis is promoted in the presence of protein synthesis inhibitors, ${ }^{15}$ suggesting derepression of endogenous inhibitors; increases in cytosol calcium fluxes inhibit rather than initiate cell death as commonly occurs in lymphoid cells. ${ }^{21}$ The short lived neutrophil can therefore be regarded as primed for apoptosis, the process normally held in check at sites of inflammation by proinflammatory mediators, cytokines, and growth factors such as GM-CSF, which not only activate neutrophils, but prolong their effective life. ${ }^{22}$ Factors such as those in synovial 
fluid that promote apoptosis are therefore likely to be restoring a natural tendency, perhaps by acting on inhibitors of apoptosis. The underlying mechanism of the synovial fluid effect might therefore relate to a number of properties such as physicochemical constitution, oxidant status, cytokine or mediator effects, protease and inhibitor levels, or immunoglobulin, complement, and immune complex interactions. The considerable variability in apoptosis promoting effect demonstrated between samples of joint fluid from different patients suggests that essential physicochemical constituents are not involved. This interpretation is supported by the lack of effect on neutrophil apoptosis incurred by addition of purified hyaluronic acid. No differences were found between samples from patients taking disease modifying agents, corticosteroids (except in fresh synovial fluid samples as discussed above), or non-steroidal antiinflammatory drugs, rendering unlikely the possibility that the synovial fluid effect relates to drugs.

Promotion of synovial neutrophil apoptosis is likely to be associated with milder synovitis, and raises the possibility that neutrophil apoptosis promoting activity is a general characteristic of inflammatory exudates, designed to contribute to pathways of inflammatory resolution. Further studies are in progress to test this postulate and to identify the mechanism by which synovial fluid influences neutrophil apoptosis.

This work was generously supported by the Arthritis and Rheumatism Council for research (UK).

1 Haslett C. Resolution of acute inflammation and the role of apoptosis in the tissue fate of granulocytes. Clin Sci 1992 83: $639-48$.

2 Crockard A D, Thompson J M, McBride S J, Edgar J D, McNeill T A, Bell A L. Markers of inflammatory activation: upregulation of complement receptors CRI and CR3 on synovial fluid neutrophils from patients with inflammatory joint disease. Clin Immunol Immunopatho 1992; 65: 135-42.

3 Savill J S, Wyllie A H, Henson J E, Walport M J, Henson P M, Haslett C. Macrophage phagocytosis of aging neutrophils in inflammation: programmed cell death leads to recognition by macrophages. $f$ Clin Invest 1989; 83: 865-75.

4 Jones S T M, Denton J, Holt P J L, Freemont A J. Possible clearance of effete polymorphonuclear leucocytes from synovial fluid by cytophagocytic mononuclear cells: implications for pathogenesis and chronicity in inflammatory arthritis. Ann Rheum Dis 1993; 52: 121-6.

5 Pekin T J, Malinin T I, Zvaifler N J. Unusual synovial fluid findings in Reiter's syndrome. Ann Int Med 1967; 66: findings

6 Spriggs A I, Boddington M M, Mowat A G. Joint fluid cytology in Reiter's syndrome. Ann Rheum Dis 1978; 37: $557-60$.

$7 \mathrm{Xu} \mathbb{W}$, Firestein G S, Taetle R, Kaushansky K, Zvaifler N J. Cytokines in chronic inflammatory arthritis II. Granulocyte-macrophage colony stimulating factor in rheumatoid synovial effusions. F Clin Invest 1989; 83: 876-82.

8 Williamson D J, Begley C G, Vadas M A, Metcalf D. The detection and initial characterisation of colony stimulating factors in synovial fluid. Clin Exp Immunol 1988; 72: 67-73.

9 Alvaro-Garcia J M, Zvaifler N J, Firestein G S. Cytokines in chronic inflammatory arthritis IV. Granulocytein chronic inflammatory arthritis IV. Granulocyteof class II MHC antigen on human monocytes: a possible of class II MHC antigen on human monocytes:
role in arthritis. $\mathcal{F}$ Exp Med 1989; 170: 865-75.

10 Firestein G S, Xu W D, Townsend K, et al. Cytokines in chronic inflammatory arthritis I. Failure to detect $T$ cell lymphokines (Interleukin 2 and Interleukin 3) and the presence of macrophage colony-stimulating factor (CSF-1) and a novel mast cell growth factor in rheumatoid synovitis. $f \operatorname{Exp} M e d 1988 ; 168$ : 1573-86.

11 Bell A L, Magill M K, McKane W R, Kirk F, Irvine A E. Measurement of colony-stimulating factors in synovial fluid: potential clinical value. Rheumatol Int 1995; 14: fluid: poter

12 Williams G T, Smith C A, Spooncer E, Dexter T M Taylor D R. Haemopoietic colony stimulating factors promote cell survival by suppressing apoptosis. Nature 1990; 343: 76-9.

13 Colatta F, Re F, Polentarutti N, Sozzani S, Mantovani A Modulation of granulocyte survival and programmed cell death by cytokines and bacterial products. Blood 1992; 80: 2012-20.

14 Adachi S, Kubota M, Wakazono Y, et al. Mechanism of enhancement of neutrophil survival by granulocyte colony-stimulating factor and adenine. Exp Hematol 1993; 21: $1213-8$.

15 Brach M A, deVos S, Gus H-J, Hermann P. Prolongation of survival of human polymorphonuclear neutrophils by granulocyte-macrophage colony-stimulating factor is granulocyte-macrophage colony-stimulating factor is
caused by inhibition of programmed cell death. Blood caused by inhibition.

16 Afford S C, Pongracz J, Stockley R A, Crocker J A, Burnett D. The induction by human interleukin- 6 of apoptosis in the promyelocytic cell line U937 and human neutrophils. f Biol Chem 1992; 267: 21612-6.

17 Takeda Y, Watanabe H, Yonehara S, Yamashita T, Saito S, Sendo F. Rapid acceleration of neutrophil apoptosis by tumor necrosis factor-a. Int Immunol 1993; 5: 691-4.

18 English D, Anderson B R. Single step separation of red blood cells, granulocytes and mononuclear leukocytes on discontinuous density gradient of ficoll-hypaque. on discontinuous density gradien

19 Irvine A E, Morris T C M, Kennedy H, Wisdom G B, Bridges J M. Human umbilical cord conditioned medium Bridges J M. Human umbilical cord conditioned medium 1984; 12: 19-24.

20 Freemont A J, Denton J. Disease distribution of synovial fluid mast cells and cytophagocytic mononuclear cells in inflammatory arthritis. Ann Rheum Dis 1985; 44: 312-5.

21 Whyte M K B, Hardwick S J, Meagher L C, Savill J S Haslett C. Transient elevations of cytosolic free calcium retard subsequent apoptosis in neutrophils in vitro. $f \mathrm{Clin}$ Invest 1993; 92: 446-55.

22 Lee A, Whyte M K, Haslett C. Inhibition of apoptosis and prolongation of neutrophil functional longevity by prolongation of neutrophil functional longevity by 